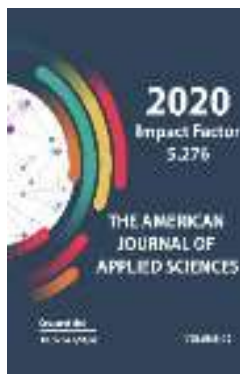

\title{
Optimization Of Heat And Mass Exchange Processes In Processing A Cell Of Oil Crops
}

\author{
U.A.Saidmuratov \\ Candidate Of Technical Sciences, Associate Professor, Department Of Machinery And \\ Equipment For Food And Chemical Industries, Bukhara Engineering-Technological Institute, \\ Bukhara, Uzbekistan
}

\begin{abstract}
Journal Website: http://usajournalshub.c om/index,php/tajas

Copyright: Original content from this work may be used under the terms of the creative commons attributes 4.0 licence.
\end{abstract}

\section{ABSTRACT}

One of the promising areas in the technology of oilseed processing is electro physical processing methods. The use of electrophysical processing methods dramatically accelerates the flow of processes, increases labor productivity, reduces the need for production facilities, and in some cases reduces energy consumption. Based on the foregoing, it is possible to formulate the main goal of studying the process of heat treatment of cotton seed mint in a two-phase flow: to identify rational conditions for the process, to outline rational ways for effective structural design of the apparatus for heat treatment of cotton seed mint.

Achieving this goal should ensure a reduction in the energy and material consumption of the process, an increase in the yield of black oil, an improvement in the quality indicators of the final products and working conditions of the staff. The analysis of technological processes occurring in a single biological cell of peppermint of cotton seeds is important, among other things, because it allows us to outline ways of choosing and synthesizing optimal process parameters, and to develop highly efficient plants.

However, the solution to the problems of optimization of continuous heat and mass transfer (HMT) processes occurring in apparatuses or installations as a whole as L-optimization is labor intensive, since when considering complex HMT systems, the number of optimizing factors increases. In the systems under consideration, the number of main factors exceeds 20 , even if they vary at two levels, more alternative options are required for optimization.

The functional decomposition of optimization problems was carried out based on the hierarchical structure of the HMT systems, in which the hydrodynamic structure of the interacting flows is considered as the main central subsystem-processes, which are subsequently divided into more than elementary heat and mass transfer (HMT) processes. Optimization of the lower levels of technological plants for the implementation of solid-state processes is considered for IR - roasting in a solvent medium (IR-LCMSR). 


\section{KEYWORDS}

Cotton seeds of cotton seeds, biotechnological phenomena, infrared heating, moisture treatment, degree of denaturation, transform, micro level, macro level, infrared irradiation.

\section{INTRODUCTION}

The main task of the modern stage of development of the food industry is the intensification of technological processes and ensuring high quality products. It is achieved through the widespread introduction in the national economy of fundamentally new technologies for processing raw materials, including: processing with pulsed and thermoradiation energy supply, taking into account the peculiarities of biotechnological phenomena that occur in the cellular structure of the processed product, which can significantly increase production productivity, increase the efficiency of resource use and reduce energy and material consumption of installations.

The process of heat treatment of oil-containing materials is one of the main stages of the technology for the production of vegetable oils, which significantly affect the quality, cost of production, working conditions of staff and the possibility of creating continuously operating mechanized lines. Therefore, the task of researching and developing methods for intensifying the process of moisture and heat treatment of oil-containing materials by improving the structure of flows is of particular relevance.

One of the most common methods of heat treatment of cottonseed mint is the roasting process, which is carried out in the fry pots with blank steam and in infrared conveyors - energy supply, which contributes to the efficient extraction of oil.

As a result of further study of the oil extraction process, a method of direct extraction of an oil- containing material arose, which excludes the process of thermal and thermal treatment of the latter. The disadvantage of this method is that during direct extraction, the peppermint of cotton seeds does not have enough in hidden cells, which makes it difficult to achieve maximum oil removal from the pulp and, therefore, there is an increased content of the target product in the meal.

At present, new progressive methods are being developed for extracting oil from oilcontaining materials in the field of electromagnetic waves of the IR range, which are free from the mentioned disadvantages [1].

In recent years, a number of studies have been carried out in our region and abroad aimed at the use of short-wave infrared emitters for frying oil-containing materials (beans, cocoa, peanuts, almonds, etc.), and testifying to the prospects of this method of heat supply for processing food and oil-containing materials.

The results of studies by the authors of $[2,9,10,16,17]$ give an idea of the mechanism of internal heat and mass transfer in the process of heat treatment (frying, drying) of food products with infrared rays and allow us to recommend optimal technological conditions.

At the same time, the heat treatment process of oil-containing materials in the fry pots does not provide an equal distribution of moisture and heat in the bulk of the material, and processing in conveyors due to infrared energy supply does not provide the required quality of the frying process. A long processing process leads to overcooking of the oil-containing 
material and other negative consequences, which significantly affect the output and quality of the oil.

Undoubtedly, the most rational way to increase the efficiency of heat treatment plants is to improve, intensify and optimize the processes of processing oil-containing materials, which is of great social importance.

The use of infrared heating and the study of the hydrodynamic structure of flows during the heat treatment of the mint of cotton seeds is one of the main ways to reveal the hidden reserves of the intensification of the process.

The penetration of infrared rays into the thickness of materials and products, as well as the specific effects of infrared radiation on their structure, are important. Along with this, the ability to control the spatial distribution of the radiant flux allows the directed heating of only the treated object, minimizing the loss of energy on heating the surrounding surface. In turn, the penetration of rays into the thickness of the processed material significantly affects the nature of the temperature and humidity distribution fields, which determines the rate of destruction of fiber (the rate of biochemical reaction), the consequences of which affect the quality of the resulting oil. Therefore, the irradiation of oil-containing raw materials with infrared rays should be considered not only as a method of intensive heat treatment, but also as a process of deep impact on the physicochemical nature of the material.

The rationale for the optimal heat treatment of mint cotton seeds, which allows to reduce the duration of the process, reduce energy consumption and improve the quality of the finished product, is essential for the industrial production of vegetable oils.

All this testifies to the particular urgency of the task of intensifying the technological process of heat treatment of cottonseed mint, based on the use of progressive methods of energy supply using the latest advances in theory to the practice of heat and mass transfer.

The main objective of the current stage of development of the food industry is the intensification of technological processes and ensuring high quality products through the widespread introduction of fundamentally new technologies for processing raw materials, including processing with pulsed and thermo-radiation energy supply, taking into account the characteristics of biotechnological processes that occur in the cellular structure of the processed product and allow to increase labor productivity, increase the efficiency of resource use and reduce the energy and material consumption of plants.

Moisture and heat treatment - preparation of oilseed peppermint for pressing and extraction - is one of the most important technological operations in the production of vegetable oils, which significantly affects the quality and cost of production, working conditions of staff. The use of non-traditional methods of heat treatment, in particular, infrared (IR) processing of mint cotton seeds allows to reduce the duration of the process, increase the yield of black and refined oil, and improve the quality of the final product. In this regard, the further intensification of the process of IR heat treatment of cotton seed mint in a twophase flow with the prevention of oxidation of the constituent components, the identification of rational processing regimes and the creation of a plant that improves the structure of material flows is an urgent task.

\section{MATERIALS AND METHODS}

Intensification of the process of roasting cotton mint in the thermo-radiation field of infrared radiation. Elimination of the oxidation of oil components by conducting a process in an inert oxygen-free environment, organizing the frying process with an optimal hydrodynamic flow. 
Analysis of methods and installations of the process of frying oil-containing materials; experimental study of the effectiveness of the influence of parameters on the frying process; development of a new method for heat treatment of cotton peppermint by IR radiation, development of the design of the installation; compilation of a mathematical model of the process of heat treatment of the mint of cotton seeds based on experimental studies; study of the process on a mathematical model and identification of optimal values of the parameters that affect the process; calculation and experimental determination of mint processing parameters; drawing up a methodology for the engineering calculation of an industrial design of a plant design for heat treatment of a peppermint with infrared energy supply and substantiation of specific recommendations; calculation of technical and economic indicators of the new installation; discussion of the technical and economic efficiency of the installation.

Oilseeds are complex heterogeneous biologically active systems; their massmoisture exchange and thermodynamic characteristics is a function of the chemical composition, structure, state parameters (density, temperature, humidity, etc.) and depend on the processing methods. Therefore, these characteristics during technological processes vary widely.

A significant portion of the processed food products are wet dispersed systems. A decisive influence on the bond of moisture with a dry skeleton and on its mass transfer characteristics is exerted by the dispersion of the material. The greater the dispersion of the material (its external and internal specific surface), the more it is firmly bound moisture. At the same time, the radius of capillaries is of great importance for porous materials: the lower the vapor pressure with the meniscus of the liquid in the capillary and the more energy must be expended to remove moisture [3].
Food products are systems in which moisture has various forms of bonding with a solid skeleton. A number of researchers simplifies the classification of moisture bonding forms and suggests distinguishing only two main groups; free and bound water.

In colloidal systems, free water corresponds to the first phase of the mechanism of interaction of water with a colloid and is an "intermicellar" liquid with known water properties. Bound water, which is especially strongly adsorbed on the surface of miscells, corresponds to the second and third phases of this process and has a number of features: it evaporates more difficult, is a poor solvent and can be under increased pressure due to the molecular force field. Therefore, as indicated above, the density of adsorption-bound water may increase slightly. The strong binding of water is sometimes linked to the theory of the formation of a "solid solution" with "intmicellar" interaction [3].

a) if the partial pressure of steam at the surface of the material is greater than the partial pressure of steam in air, then the evaporation process (desorption) occurs;

b) if, then the material is moistened as a result of the absorption of steam from the ambient air (sorption).

When moistening (sorption), deep structural changes of oilseeds occur due to physicochemical differences in hydration and swelling of the structural elements of the mint (protein formations, fiber, etc.), which leads to a volumetric stress state and the formation of microcracks. As a result of this, during sorption, the inner surface on which the adsorption binding of water occurs increases. Therefore, during subsequent desorption, more bound moisture must be removed.

The practical interest is presented a comprehensive study of the forms of bonding and the state of moisture, as well as the binding energy of moisture in colloidal 
capillary-porous materials. As colloidal capillary-porous bodies, we consider oilcontaining products (cotton seeds).

With moisture-thermal treatment, biochemical changes occur in the mint of cotton seeds. The total effect of moisture, heat, and oxygen during frying promotes activation of the peppermint enzyme system, which leads to the intensive occurrence of undesirable hydrolytic, proteolytic, and oxidative processes in it [11].

The mint subjected to frying has a very complex enzyme composition - different for seeds of various oilseeds. Peppermint contains the entire set of enzymes of live seed; therefore, a complex of biochemical processes takes place in it, the course of which has a significant impact on the quality of the final products of the processing of oilseeds.

Exceeding the humidity and temperature of the peppermint of cotton seeds at the level of maximum permissible values at the beginning of the frying process leads to an increase in the activity of the enzyme system, and further heating leads to a decrease in the activity of the enzymes. These properties of the enzyme system are widely used in industry to ensure the production of high-quality oil. However, due to the long duration of the frying process, it is not always possible to obtain high-quality oil.

The degree of denaturation of peppermint proteins of cotton seeds and other seeds depends on the frying mode. In [14], it was pointed out that with an increase in the initial moisture of the mint and the temperature of the pulp, the degree of denaturation of protein substances steadily increases.

The mint of cotton seeds differs from others in that it contains a coloring matter - gossypol. The content of gossypol in the nucleus ranges from to the mass of the nucleus.

Under the influence of heat, moisture, oxygen in the process of roasting the mint of cotton seeds, various transformations of gossypol occur.

The increased humidity and temperature of the mint of cotton seeds, as well as certain duration of heat exposure facilitate the dissolution of gossypol in oil.

Intensive moisture - heat treatment leads to the accumulation of gossyprotein compounds in the pulp, a decrease in lysine content compared to the mild regimen, in which gossypol passes into oil [15].

From the foregoing, it can be concluded that the frying process is a complex set of physical and chemical changes in the mint. It is necessary to conduct further research aimed at intensifying the process and improving the quality of the products obtained.

The preparation of pulp (frying) is an operation consisting in treating the mint with heat and water for a certain period of time with stirring in special roasting apparatus.

The purpose of this operation is to cause certain physicochemical changes in the mint and transform the structure of its particles in order to contribute to the best effect of further oil extraction. In addition, in some cases, when frying, it is necessary to carry out additional chemical changes to ensure the highest quality products (for example, in the processing of cotton seeds - binding of gossypol, soybean seeds - changing some proteins and removing unpleasant smelling substances with water vapor).

Food technology processes are complex physicochemical systems having a dual deterministic stochastic nature, the nature of which is variable in space and time in parameters. The substance flows participating in them are, as a rule, multiphase and multicomponent. The whole process as a whole proceeds in an apparatus with specific geometric characteristics, which, in turn, have an effect on the nature of the ongoing process. 
The dissipative function (local production of entropy) characterizes all types of energy consumption for the course of irreversible processes in the system. The decomposition of the main driving force into components is given in [5].

When modeling solid-state processes that are complicated by physicochemical transformations, an important step in the qualitative analysis is the discovery of the driving forces of the processes.

When analyzing the processes occurring in technological devices, it is customary to conventionally divide the entire set of physicochemical phenomena and effects that occur in them into two levels: microlevel and macrolevel [5]. Microkinetic factors include a set of physicochemical effects that determine the rate of occurrence of biological, physical or chemical phenomena at the molecular, cellular level and in the local volume of apparatus flows. The macrokinetics of the process studies the behavior of the process on the scale of the apparatus as a whole. Here, hydrodynamic, thermal, diffusion phenomena of a large-scale nature are superimposed on the effects of the microlevel.

It is usually difficult to draw a sharp line between effects and phenomena occurring at the micro and macro levels. In this regard, it becomes necessary to introduce intermediate levels of phenomena and effects.

Mathematical modeling of chemicaltechnological processes, acad. V.V. Kafarov, should be carried out interconnectedly between three stages: formalization of the process under study - construction of the mathematical model proper; algorithmization, providing the numerical values of certain parameters; and establishing the adequacy of the model that describes the real process.

The multidimensionality of HMTR leads to significant simplifications of the generalized description, which complicates the maintenance of a reasonable degree of detail of the mathematical description.

These circumstances determine the third approach to the synthesis of the mathematical model of HMTR, based on model concepts of internal biochemical transformations occurring in technological devices. The basis of this approach is a set of elementary models that reflect the simplest physicochemical phenomena (ideal mixing model, ideal displacement model, diffusion model, cell model, combined models, etc.). Obtaining a mathematical description of HMTR in this case comes down to the selection of such a combination of typical models so that the resulting model accurately reflects the basic laws of biochemical transformations of the existing and synthesized HMTR. This approach makes it relatively easy to take into account the influence of the most important factors in the system at the macro level, as well as the stochastic properties of the HMTR.

The construction of a complete mathematical model of HMTR is completed by the aggregation of individual subsystems or blocks (hydrodynamics block, biochemical block, chemical kinetics block, diffusion kinetics block, thermal phenomena kinetics block, equilibrium relations block, etc.) into a single system.

With this, the verification of the adequacy of the mathematical model of HMTR and the identification of its parameters is performed on the basis of the theory of solving inverse problems of mathematical physics, identification and estimation of state parameters of systems. Since the first two stages usually make it possible to synthesize the structure of the mathematical model of the HMTR that is quite close to the physical structure of the HMTR, the identification task in the third stage is to search for unknown model parameters based on a given criterion for the correspondence of experimental and calculated data $[5,8]$. 
Mathematical modeling and optimization, physico-chemical research methods, multifactor design of experiments.The use of system analysis methods to solve the problems of studying the processes of industrial processing of oilseeds provides an opportunity to take into account all the most significant factors affecting the adjacent technological processes of oil-fat production.

The essence of system analysis and the general principles applied to the solution of any system problem are adequately reflected in the works of academician V.V. Kafarov, prof. Dorokhova I.N. $[5,6,7,8]$.

A qualitative analysis of the structure of HMTR includes semantic (that is, a preliminary analysis of a priori information on the physicochemical, biochemical, biophysical, and other features of HMTR) and mathematical (that is, a qualitative analysis of the structure of mathematical dependencies included in the description of HMTR).

Consideration of the structure of material, heat and other flows in a HMTR as its main subsystem allows us to more correctly represent the complex internal structure of processes.

To build a hierarchical structure and implement the first stage of a semantic analysis of processes, it is necessary to separate the processes of heat and mass transfer that occur simultaneously with processes and moisture treatment. The construction of the hierarchical structure of the object of study allows us to reveal the relationship between the phenomena occurring in physicochemical processes, to obtain their mathematical descriptions at the hierarchy levels, starting from the atomic-molecular and, of course, processed layer of the product processed in the apparatus under consideration.

In connection with the foregoing and according to the general principles of system analysis, we determine the hierarchical structure of the main PSTNs. At the same time, we consider each of them as an independent system.

It is advisable to consider a combination of chemical, physico-chemical, biochemical, biophysical, heat transfer, heat and mass transfer phenomena and effects that take place in a HMTR at separate steps in the hierarchy of heat and mass transfer processes. Moreover, the number of hierarchy levels of each system is determined by the complexity of its structure.

The following is a qualitative analysis of the processes occurring in the subsystems of the hierarchical structure of PSTN. At the same time, in each hierarchical stage of the corresponding HMTR, its own subsystem is determined with the corresponding main input and output parameters characterizing the behavior of this subsystem, ways of managing and improving the organization of processes in the HMTR subsystems are considered.

The formulation of the optimization problem includes the selection of an optimality criterion, the establishment of constraints, the selection of optimizing factors, and the determination of the objective function [4].

At the second level, the object of optimization for the HMT of the IR-LCMSR process is an oilcontaining cell. For the cases under consideration, the main targeted elementary process is the maximum destruction of the wall of oil-containing cells, which ultimately contributes to an increase in oil yield and intensifies mass transfer in the solid phase. Therefore, as a criterion of optimality, the degree of destruction of the wall of the oilcontaining cell is selected, and the limitations are the density of the infrared flow temperature, humidity, irradiation and exposure time and other parameters characterizing the properties of the components of the material, as well as quality 
indicators of the resulting products. Of these, optimizing factors can be distinguished.

At the third level, the objects of optimization are:

- in IR-GHMSR - a particle of a solid phase. The degree of frying of the particles of oilcontaining material is characterized by the number of destroyed cells in it. Therefore, the degree of destruction of the cell walls in a particle of an oil-containing material is selected as an optimality criterion. The limitations in IRGHMSR are pipe diameter, suspension and miscella concentration, IR flow density, temperature, humidity, exposure time and exposure. Optimizing factors in IR-LCMSR are: pipe diameter and suspension and miscella concentration.

At the fourth level of optimization, the object of elementary HMT processes is the hydrodynamic structure of flows. The processes occurring in the particles of the streams are indirectly characterized by the hydrodynamic situation in the apparatus. From the point of view of analysis and synthesis of HMT processes, it is most expedient to fix the structure of flows by a cellular model. Therefore, the number of cells is selected as a criterion for the optimality of the considered HMT processes. Limitations are design indicators and parameters characterizing the hydrodynamic conditions of the process (etc.). Optimizing factors are selected in IR-LCMSR the ratio of the length of the pipe to its diameter.

\section{RESULT AND DISCUSSION}

At the fifth level, the objects of optimization are devices or an installation for the implementation of solid-state processes. Here, the optimization problem can be formulated as a multicriteria problem. At this level, optimization objects are characterized by structural and technological indicators. The following criteria are accepted as optimization criteria: for the implementation of IR-LCMS in tubular installations (TU) - the working surface, temperature, processing time and energy consumption.

In this regard, the optimization problem can be formulated in the form of the following mathematical expressions:

$$
\begin{aligned}
& F=f(G, u, q, \tau \ldots) \rightarrow \min \\
& t=f(G, u, q, \tau \ldots) \rightarrow t_{\mathrm{spec}} \\
& \tau=f(G, u, q, \tau \ldots) \rightarrow \min \\
& \ni=f(G, u, q, \tau \ldots) \rightarrow \min
\end{aligned}
$$

The minimum values are determined by experimental and theoretical studies $[12,13]$ at the lower levels of optimization, providing a given temperature of the final product, resulting from the requirements of subsequent technological processes.

Based on the foregoing, the optimization problem can logically be reduced to the following form:

$$
F=\rightarrow f(L) \rightarrow \min
$$

The limitations for this problem are the corresponding mathematical models that connect the input and output parameters of the process and $\mathrm{G}, \mathrm{u}, \mathrm{t}, \mathrm{q}, \tau$. In this case, and are chosen as optimizing factors.

At the sixth level, the objects of optimization are installations for IR-LCMS. The objects under consideration are part of a production line in which intermediate products are obtained. Therefore, as an economic criterion of optimality in this particular case, it is advisable to choose the sum of variable and fixed costs, referred to the unit of output. In accordance with this, the optimization problem for each installation can be expressed by the following mathematical expression:

$$
3=f\left(E, G_{y}, K_{a} P \ldots\right) \rightarrow \min
$$


At the same time, the constraints on the objective function are distinguished in the form of equalities - mathematical models of the main and auxiliary apparatuses of the installation, which interconnect input and output parameters and in the form of inequalities - the restrictions imposed on electricity consumption, plant performance, and product quality indicators. The following optimizing factor can be distinguished: power consumption.

In the considered problems of optimization of HMT systems in the lower I-IV levels, the types of objective functions are unknown. Therefore, to find the extremum of the objective functions at these levels, the experimental method is used.

At the fifth level of optimization, due to the difficulty of reducing the expression of objective functions to explicit types and the difficulty of differentiating, due to its multifactorial nature, the possibility of using analytical optimization methods is excluded. Since at this level of the hierarchy, the objective function is given by calculation algorithms for given values of factors, and the mathematical models of solid-state processes are constructed according to the block principle with structuring according to the flow structure. For such cases, it is advisable to use numerical optimization methods. To solve the classes of problems under consideration, it is easiest to apply optimization by enumeration and scanning [4].

Solving optimization problems at the first level of the hierarchy. The task of the molecular level is to determine the thermophysical parameters and constants of biochemical reactions.

The coefficients of biochemical reactions are determined experimentally by solving the inverse problem for the optimal management of IR-LCMSR.

They have the following meanings:

$$
\kappa_{01}=9,9 s^{-1}, \kappa_{02}=0,000124 s^{-1}, \kappa_{1}=0,003219 s^{-1}, \kappa_{2}=0,0004 s^{-1}, \kappa_{3}=0,003219 s^{-1} \text {. }
$$

The solution to optimization problems is the second level. The degree of destruction of the wall of the oil-containing cell is characterized by the amount of decomposition of polysaccharides into di- and monosaccharides. The formation of monosaccharides was experimentally determined by the author of Ref. [12] by the Orthotluid and HagedornJonsana methods. The obtained experimental data confirm the reliability of the conversion from the total mass of the nucleus of the cell walls to monosaccharides, which is equal to the total mass of fiber. According to the conclusions based on experimental data [12], during the breakdown of polysaccharides, a complete destruction of the fiber wall is achieved, thereby maximizing the oil yield.
Methods of planning experiments determined the values of influencing factors that provide the maximum oil yield, respectively, the maximum degree of destruction of the fiber wall, which have the following values for IRGHMSR $\quad q=450 \kappa V t / \mu^{2}, \quad \tau_{1}=1 s \quad$ и $\tau_{2}=240 s$ under restrictions $4 \leq u \leq 9 \%$, and $60 \leq t \leq 80^{\circ} \mathrm{C}$

Problem solving at the third level of optimization. According to the results of experimental studies, it was found that with IRGHMSR, the maximum destruction of the cell wall in the mint particle $\left(r_{\mathrm{p}} \rightarrow 100 \%\right)$ provided when $\quad d_{T P}=50 \mathrm{MM}, \quad a_{m i s}=10 \% \quad$ and $a_{s}=27 \%$. 
Algorithmization and problem solving of the fourth level of optimization. The determination of the minimum length of the IR - irradiation zone during IR-LCMSR is carried out according to an algorithm that implies the following sequence of actions:

1. Initial parameter values are set: $G_{\text {mint }}, a_{s}, \rho_{s}, d_{\text {pip }}$

2. Suspension flow is determined $G_{c}=\frac{G_{\text {mint }} \cdot 100}{100-a_{c}}$

3.

The volumetric flow rate of the

$$
V_{s}=\frac{\boldsymbol{G}_{s}}{\boldsymbol{P}}
$$

suspension is calculated.
4

The average suspension rate in the pipe is determined.

$$
W_{s}=\frac{4 V_{s}}{\pi d_{p i p}^{2}}
$$

5. Using the experimentally determined value of the duration of IR exposure, the pipe length is calculated

$$
L_{\min }=W_{s} * \tau
$$

\begin{tabular}{|c|c|c|c|c|c|}
\hline $\begin{array}{l}\text { Optimiza } \\
\text { tions }\end{array}$ & $\begin{array}{c}\text { Process class } \\
\text { and level } \\
\text { object }\end{array}$ & $\begin{array}{l}\text { Optimization Criteria } \\
\text { (Goal Function) }\end{array}$ & $\begin{array}{l}\text { Optimizing } \\
\text { factors }\end{array}$ & Limitations & $\begin{array}{c}\text { Optimal parameter } \\
\text { values }\end{array}$ \\
\hline Apparatus & $\begin{array}{c}\text { Tubular } \\
\text { installation }\end{array}$ & $R(L)=\frac{4 * \frac{\frac{G_{\text {mpzu }} * 100}{100-a_{c}}}{\rho}}{\pi * D^{2}} * \tau$ & $\mathrm{q}, \tau$ & $\begin{array}{c}G_{\text {mivt }}=0,2 \mathrm{kdh} \\
4 \leq u \leq 9 \% \\
60 \leq t \leq 80^{\circ} \mathrm{C}\end{array}$ & $L_{m p m \min }=0,4 m$ \\
\hline Molecule & $\begin{array}{l}\text { Cellulose } \\
\text { protein, } \\
\text { etc. }\end{array}$ & \multicolumn{4}{|c|}{ The coefficients of biochemical reactions } \\
\hline & & \multicolumn{4}{|c|}{$k_{01}=9.90 s^{-1}, k_{02}=0,0001 s^{-1}, k_{1}=0.0032 s^{-1}, k_{2}=0.0004 s^{-1}, k_{3}=0.0032 s^{-1}$} \\
\hline
\end{tabular}

Table 1 - the optimal values of the modal and process control

According to the above algorithm, the minimum pipe length of the IR-irradiation zone for IR-LCMSR was determined for the following initial data: $G_{\min t}=0.2 \mathrm{~kg} / \mathrm{s}, \quad a_{s}=27 \%$, $\tau=1 s$, which is $L_{\text {min }}=0.4 m$.
On a unified methodological basis, the formalization of multilevel and multicriteria optimization problems for solid-state processes is carried out. The selection of optimization methods and the algorithmization of the solution of objective functions are carried out. 


\section{CONCLUSION}

The optimal structural and technological parameters of the installation for the implementation of the process of infrared roasting of cotton mint in a solvent are determined. The duration of active exposure to high temperatures was reduced by more than 3,000 times with IR-GHMSR compared with the duration of exposure to high temperatures during traditional frying in bath roasters. Improving the quality of products through the implementation of developments is confirmed by the conclusions of laboratories.

The questions of chemical transformations are treated in the treatment of mint cotton seeds in a solvent medium in the field of IR - radiation.

As a result of theoretical and experimental studies, a mathematical model of the process of thermal radiation treatment of mint cotton seeds in a solvent environment is obtained. An algorithm for solving a mathematical model is developed, software implemented in the MATLAB algorithmic language as applied to

IBM PC. The basic laws of the heat treatment of mint cotton seeds in an organic solvent are investigated.

\section{REFERENCES}

1. A. p. 13677471 . (USSR), $M K / 3$ C $11 \mathrm{~V} 1 / 10$. A method of producing cottonseed oil / Artikov A.A., Usmanov A.U., Mamatkulov A.Kh. et al. (USSR). - No. 3894705/31 - 13; Application 01.04.85; Publ. 09/15/87, particleboard. - 5 p.: Ill. UDC 665.1 (088.8).

2. Ginzburg A.S. Infrared technology in the food industry. $-M$.: Food industry, 1973. $408 \mathrm{p}$.

3. Ginzburg A.S., Savina I.M. Mass and moisture exchange characteristics of food products. - $M$.: Light and food industry, 1982. -279 p.
4. Zakheim A.YU. Introduction to the modeling of chemical - technological processes. - $M$.: Chemistry, 1982. - 288 p.

5. Kafarov V.V., Dorokhov I.N. System analysis of chemical technology processes. - $M$.: Science. 1976. - $500 \mathrm{p}$.

6. Kafarov V.V., Dorokhov I.N. System analysis of chemical technology processes. The topological principle of formalization. - $\mathrm{M}$.: Science. 1979. - $400 \mathrm{p}$.

7. Kafarov V.V., Dorokhov I.N., Lipatov L.N. System analysis of chemical technology processes. - M .: Nauka, 1982.- 344 p.

8. Kafarov V.V., Glebov M.V. Mathematical modeling of the basic processes of chemical production.- M.: Higher School, 1991. - 400 p.

9. Kozelkin V.V., Usoltsev I.F. Fundamentals of IR - technology. - M.: Mechanical Engineering. 1974.- $336 \mathrm{p}$.

10. Rogov I.A. Electrophysical methods of food processing. - M .: Agropromizdat, 1988 .-$272 \mathrm{p}$.

11. Guides on the technology for the production and processing of vegetable oils and fats / Ed. A.G. Sergeeva. - L .: ARRIAH, T1. Prince the second, 1974.- $591 \mathrm{~s}$.

12. Safarov AF, Moisture and heat treatment of oilseeds: Dis ... doct. tech. sciences. Tashkent, 1991. -297 p.

13. Saidmuratov U.A. Optimization of heat and mass transfer process of roasting cotton mint // Chemical technology, control and management. - Tashkent, 2008. No. 6. - S. 32-34.

14. The technology of production of vegetable oils / Kopeikovsky V. M., Danilchik S. I., Garbuzova G. I. and others. - M.: Light and food industry. 1982. - $416 \mathrm{p}$.

15. Shcherbakov V.G. Biochemistry and commodity science of oilseeds. - M.: Food Industry, 1979. - $336 \mathrm{p}$.

16. Vavsko A. UK-radiation-London: Iliffe Books-Prague. Techn., Liter. 1968. 